\title{
ON THE LEAST POSITIVE EIGENVALUE OF INTEGRAL EQUATIONS WITH EQUIMEASURABLE KERNELS $\left({ }^{1}\right)$
}

BY

\section{BINYAMIN SCHWARZ}

Rearrangements of sets of numbers and rearrangements of functions were defined and investigated in detail in the book of Hardy, Littlewood and Pólya [4, Chapter X]. Using this notion, classes of nonhomogeneous strings, membranes, rods and plates with equimeasurable density were considered by P. R. Beesack and the author and the extrema of the principal frequency were found for these classes $[1 ; 2 ; 7]$. Here we deal with an analogous question for integral equations. For any equimeasurable class of non-negative and symmetric $L^{2}$ kernels we find the maximum of the first eigenvalue. The proof is elementary and uses only the maximum property of the reciprocal of the first eigenvalue $[3 ; 8 ; 9]$ and a simple geometric idea. After the proof of this theorem we add some remarks on the corresponding minimum and on the same problem for matrices.

TheOREM. Let $K(x, y)$ be a real and symmetric $L^{2}$ kernel $\left({ }^{2}\right)$ in $0 \leqq x \leqq a$, $0 \leqq y \leqq a,(0<a<\infty)$ taking there only non-negative values (but $K(x, y)>0$ in a set of positive measure). Denote the class of all symmetric kernels $M(x, y)$ which in the above square are equimeasurable to $K(x, y)$ by $E(K)$. Let $\lambda_{1}(M)$ be the least positive eigenvalue of the integral equation

$$
\phi(x)=\lambda \int_{0}^{a} M(x, y) \phi(y) d y, \quad 0 \leqq x \leqq a .
$$

Then

$$
\operatorname{Max}_{M \in E(K)} \lambda_{1}(M)=\frac{1}{|K|},
$$

where

$$
|K|=\frac{1}{a} \int_{0}^{a} \int_{0}^{a} K(x, y) d x d y .
$$

Received by the editors July 31, 1961 .

(1) The research reported in this document has been sponsored in part by the Air Force Office of Scientific Research of the Air Research and Development Command United States Air Force through its European Office, under Contract No. AF 61(052)-323.

(2) As usually defined, see e.g. [9]. Smithies [8] calls these kernels $L^{2}$ kernels in the wide sense. The kernel $K^{*}$ which we construct below will be a $L^{2}$ kernel in the strict sense of [8]. 
Proof. We divide the proof into three parts. The decisive step is the last one, but for completeness we give also the details of the well-known first two parts.

First, it follows from the maximum property of $\kappa_{1}(M)=\lambda_{1}(M)^{-1}$ that for each $M \in E(K)$

$$
\lambda_{1}(M) \leqq \frac{1}{|K|}
$$

Indeed, using the notation

$$
J_{M}(\phi, \phi)=\int_{0}^{a} \int_{0}^{a} M(x, y) \phi(x) \phi(y) d x d y,
$$

we have

$$
\begin{aligned}
\frac{1}{\lambda_{1}(M)} & =\kappa_{1}(M)=\operatorname{Max} J_{M}(\phi, \phi) \geqq J_{M}\left(a^{-1 / 2}, a^{-1 / 2}\right) \\
& =\frac{1}{a} \int_{0}^{a} \int_{0}^{a} M(x, y) d x d y=|K| .
\end{aligned}
$$

The maximum in the third term is taken over all real normalized $L^{2}$ functions $\phi(x)\left(\int_{0}^{a} \phi^{2} d x=1\right)$. The last equality sign follows from the equimeasurability of $M$ and $K$. This proves (4). Cf. [9, p. 121].

The second step consists in the following lemma which is known in much more general circumstances and goes back to Jentzsch [5] (see also Karlin [6]).

Lemma. A ssume that the constant $a^{-1 / 2}$ is a (normalized) eigenfunction $\phi(x)$ of the kernel $K^{*} \in E(K)$. Then the corresponding eigenvalue

$$
\lambda\left(K^{*}\right)=\frac{1}{|K|}
$$

is the least positive eigenvalue $\lambda_{1}\left(K^{*}\right)$ of $K^{*}$.

The equality sign in (5) follows from

$$
\frac{1}{\lambda\left(K^{*}\right)}=J_{K^{*}}\left(a^{-1 / 2}, a^{-1 / 2}\right)=|K| .
$$

To show that this eigenvalue $\lambda\left(K^{*}\right)$ is the least positive eigenvalue $\lambda_{1}\left(K^{*}\right)$ of $K^{*}$, we use that $K^{*}$ has a (normalized) non-negative first eigenfunction $\Phi_{1}(x)$. Indeed, let $\phi_{1}(x)$ be any normalized first eigenfunction. $K^{*}(x, y) \geqq 0$ implies

$$
J_{K^{*}}\left(\left|\phi_{1}\right|,\left|\phi_{1}\right|\right) \geqq J_{K^{*}}\left(\phi_{1}, \phi_{1}\right) \text {. }
$$

The maximum property of $\lambda_{1}\left(K^{*}\right)^{-1}$ implies that equality holds in (6) and 
the maximum characterization of the first eigenfunctions gives now that together with $\phi_{1}(x)$ also $\Phi_{1}(x)=\left|\phi_{1}(x)\right|$ is a first eigenfunction of $K^{*}$. Eigenfunctions belonging to different eigenvalues are orthogonal, but $\Phi_{1}(x) \geqq 0$ and $\phi(x)=a^{-1 / 2}$ cannot be orthogonal and we thus proved the lemma.

We come now to the last step in the proof of the theorem. We show that given any $K$, fulfilling the assumptions of the theorem, there exist always kernels $K^{*} \in E(K)$ for which the assumption of the lemma holds. For the proof it will be enough to build one such kernel $K^{*}$.

The assumption of the lemma was that $\phi(x)=a^{-1 / 2}$ is an eigenfunction of $K^{*}$. As $K^{*}$ has to be equimeasurable to $K$, this is equivalent to

$$
\int_{0}^{a} K^{*}(x, y) d y=|K|, \quad 0 \leqq x \leqq a\left({ }^{3}\right) .
$$

Given $K$, we shall construct $K^{*} \in E(K)$ so that not only (7) is fulfilled but even a stronger condition; i.e., we shall achieve not only that the integrals with regard to $y$ are independent of $x$ but that the functions $g_{x}(y)=K^{*}(x, y)$ are, for all values of the parameter $x$, equimeasurable one to another in $0 \leqq y \leqq a$.

To clarify the geometric idea of the construction we consider first a simple special case, namely a function $K(x, y)$ taking only the values 0 and 1 . Let $\alpha a^{2}, 0<\alpha<1$, be the measure of the set where $K(x, y)=1$. For the construction of $K^{*}$ we cut the square $0 \leqq x \leqq a, 0 \leqq y \leqq a$ by the following four parallels to its main diagonal: $x-y= \pm \alpha a / 2$ and $x-y= \pm a(1-\alpha / 2)$. The square is thus divided into five subsets. We define $K^{*}$ by letting it take the value 1 in the two triangles and in the middle set (containing the main diagonal) and taking the value 0 in the two remaining sets. Clearly $K^{*} \in E(K)$ and on every segment parallel to the $y$-axis $K^{*}$ takes the value 1 in a set of measure $\alpha a$.

For a general (symmetric and non-negative $L^{2}$ ) kernel $K(x, y)$ we build $K^{*}(x, y)$ by the same method. Let $m(u), u \geqq 0$, be the measure of the set (in the square) in which $K(x, y) \geqq u . m(u)$ is a decreasing function of $u, m(0)=a^{2}$, $m(\infty)=0$ and $m(u)$ characterizes the class $E(K)$. Following [4] we define the inverse $\psi$ of $m$ by $\psi(m(u))=u . \psi(z)$ is a decreasing function of $z$ defined uniquely in $\left[0, a^{2}\right]$ except for at most a countable set of values $z$ corresponding to intervals of constancy of $m(u)$. We complete the definition of $\psi(z)$ by agreeing that

$$
\psi(z)=\frac{1}{2}\{\psi(z-0)+\psi(z+0)\}
$$

at its points of discontinuity. $\psi(z)$ is the one-dimensional rearrangement of $K(x, y)$ in decreasing order. The measure of the set in $\left[0, a^{2}\right]$ in which $\psi(z) \geqq u$

$\left(^{3}\right)(7)$ has to hold only for almost all $x$; we shall however construct $K^{*}$ so that it does hold for all $x$. See also footnote 2 . 
is $m(u)$; indeed this set is an interval whose left end-point is $z=0$ and whose right end-point $z=m(u)$ may or may not belong to the set. Let $k(z)=\psi(2 a z)$. $k(z)$ is defined and decreasing in $[0, a / 2]$ and $k(z) \geqq u$ in the interval with endpoints at $z=0$ and $z=m(u) / 2 a$.

For $-a \leqq s \leqq a$ we define the function $\{s\}$ by

$$
\{s\}=\left\{\begin{array}{cl}
|s| & \text { for } 0 \leqq|s| \leqq a / 2, \\
a-|s| & \text { for } a / 2 \leqq|s| \leqq a .
\end{array}\right.
$$

Using these definitions the kernel $K^{*}$ is now given by

$$
K^{*}(x, y)=k(\{x-y\}), \quad 0 \leqq x \leqq a, 0 \leqq y \leqq a .
$$

Each set $K^{*}(x, y) \geqq u(u \geqq 0)$ in the square has the same structure as the set in which $K^{*}$ of the special case was equal to 1 and this set has clearly the measure $m(u)$. Hence $K^{*} \in E(K)$.

Moreover, $K^{*}(x, y)$ has the desired property which was stated after formula (7). The graph of $g_{x^{\prime}}(y)=K^{*}\left(x^{\prime}, y\right)$ is obtained from the graph of $g_{x}(y)$ $=K^{*}(x, y)$ by a translation modulo $a$ at the amount of $x^{\prime}-x$. The functions $g_{x}(y)$ are therefore equimeasurable. This implies (7) and we thus proved the third step (i.e., the italicized statement between (6) and (7)). This and the first two steps ((4) and the lemma) complete the proof of the theorem.

Our construction of $K^{*}$ was unique. But we could take instead of the decreasing $\psi(z)$ any other one-dimensional rearrangement $\psi_{1}(z)$ of $K(x, y)$. Hence, except for the trivial case $K(x, y) \equiv c$, there are always infinitely many kernels $M$ in $E(K)$ for which $\lambda_{1}(M)$ takes its maximum. This is also implied by the following, more general, consideration.

It is natural to split each equimeasurable class $E(K)$ into "trivially equimeasurable" subclasses. Let $r(z)$ be equimeasurable to the identity function $I(z) \equiv z$ in $0 \leqq z \leqq a$. Then we say that the two kernels $M(x, y)$ and $M_{1}(x, y)$ $=M(r(x), r(y))$ are trivially equimeasurable. $M \in E(K)$ implies $M_{1} \in E(K)$. Trivially equimeasurable kernels have the same eigenvalues. Indeed, let $\lambda$ and $\phi(x)$ be an eigenvalue and a corresponding eigenfunction of $M$. (1) implies

$$
\begin{aligned}
\lambda \int_{0}^{a} M_{1}(x, y) \phi(r(y)) d y & =\lambda \int_{0}^{a} M(r(x), r(y)) \phi(r(y)) d y \\
& =\lambda \int_{0}^{a} M(r(x), y) \phi(y) d y=\phi(r(x)) .
\end{aligned}
$$

Here the second equality sign follows by the equimeasurability of $r(y)$ and $y[4] . \lambda$ is thus also an eigenvalue of $M_{1}$ (and $\phi_{1}(x)=\phi(r(x))$ is the corresponding eigenfunction). Together with the above constructed $K^{*}$ all its trivial rearrangements take the maximal $\lambda_{1}$. However, we do not claim that this maximum is attained only for one such subclass. The following example 
shows that $\operatorname{Max} \lambda_{1}(M)$ for $M \in E(K)$ may be taken in several subclasses of a given class $E(K)$ :

Let $a=1$ and set

$$
\begin{aligned}
K(x, y) & =\left\{\begin{array}{l}
1 \text { for }\{0 \leqq x \leqq 1 / 2,0 \leqq y \leqq 1 / 2\} \text { and for }\{1 / 2 \leqq x \leqq 1,1 / 2 \leqq y \leqq 1\}, \\
0 \text { for the remaining two quarters of the unit square; }
\end{array}\right. \\
K^{\prime}(x, y) & =1-K(x, y) .
\end{aligned}
$$

$K$ and $K^{\prime}$ are equimeasurable and both are kernels of rank two. The eigenvalues of $K$ are 2, 2; and of $K^{\prime}, 2,-2 . \lambda_{1}(K)=\lambda_{1}\left(K^{\prime}\right)=2=|K|^{-1}$, but the two kernels are not trivially equimeasurable, as $\lambda_{2}(K) \neq \lambda_{2}\left(K^{\prime}\right)$. (It follows also that in this case some, but not all, subclasses of $E(K)$ are positive definite.) Moreover, the corresponding $K^{*}$ is not of finite rank.

We add some remarks with regard to the minimum of $\lambda_{1}(M), M \in E(K)$. Let $\|K\|$ be the $L^{2}$ norm of $K(x, y)$; i.e.,

$$
\|K\|=\left(\int_{0}^{a} \int_{0}^{a} K^{2}(x, y) d x d y\right)^{1 / 2} .
$$

$M \in E(K)$ gives $\|M\|=\|K\|$. It is well known that

$$
\lambda_{1}(M) \geqq \frac{1}{\|M\|},
$$

and we obtain

$$
\operatorname{Min}_{M \in E(k)} \lambda_{1}(M) \geqq \frac{1}{\|K\|} .
$$

Equality holds in (10) only if $M$ is of rank one. This follows e.g. from $\|M\|^{2}$ $=\sum \lambda_{n}(M)^{-2}$; or, more elementary, by remarking that in

$$
\lambda_{1}(M)^{-2}=J_{M}\left(\phi_{1}, \phi_{1}\right)^{2} \leqq\|M\|^{2}
$$

there holds equality between the last two terms only if

$$
M(x, y)=\frac{\phi_{1}(x) \phi_{1}(y)}{\lambda_{1}(M)} .
$$

It follows that

$$
\operatorname{Min}_{M \in E(K)} \lambda_{1}(M)=\frac{1}{\|K\|}
$$

if and only if the class $E(K)$ contains kernels of rank one. However, in general, $E(K)$ does not contain such kernels. For instance, if $K$ takes two positive values only, then no $M \in E(K)$ can be of the form $g(x) g(y)$ as this product 
would take either one or at least three different values. We did not find the minimum (or infimum) of $\lambda_{1}(M)$ where $M$ goes over a (general) equimeasurable class $E(K)$.

We conclude with a remark on matrices. Let $M=\left(m_{i j}\right)$ be a symmetric matrix of order $n$ with non-negative elements $m_{i j}$. Let $\kappa_{1}(M)$ be its largest eigenvalue. Using the maximum property of $\kappa_{1}(M)$ [3], we have (in analogy to (4) and (11))

$$
\|M\|_{1} \leqq \kappa_{1}(M) \leqq\|M\|_{2} .
$$

Here the norms are defined by

$$
\|M\|_{1}=\frac{1}{n} \sum_{i, j=1}^{n} m_{i j}, \quad\|M\|_{2}=\left(\sum_{i, j=1}^{n} m_{i j}^{2}\right)^{1 / 2} .
$$

Let now $E(M)$ be the class of all symmetric rearrangements of $M$; i.e., the class of all symmetric matrices whose elements are a permutation of the elements $m_{i j}$ of $M$. The norms are invariants of the class and there arises the question for which classes the bounds in (12) are attained. In analogy to the first two steps of the proof of the theorem it follows that

$$
\underset{M \in E(A)}{\operatorname{Min}} \kappa_{1}(M)=\|A\|_{1}
$$

whenever $E(A)$ contains a symmetric generalized stochastic matrix $B$ (i.e., $b_{i j}=b_{j i} \geqq 0$ and such that $\left.\sum_{j=1}^{n} b_{i j}=\|A\|_{1}, i=1, \cdots, n\right)$. This property corresponds to (7); but in contradistinction to the results for kernels, not every class $E(A)$ contains such a matrix $B$. In analogy to $\left(11^{\prime}\right)$ we have

$$
\operatorname{Max}_{M \in E(A)} \kappa_{1}(M)=\|A\|_{2}
$$

whenever $E(A)$ contains a matrix $C$ of rank one

$$
\text { (so that } \left.c_{i j}=\gamma_{i} \gamma_{j} ; \gamma_{i} \geqq 0, \sum_{i=1}^{n} \gamma_{i}^{2}=\|A\|_{2}\right) \text {. }
$$

There exist classes $E(A)$ containing both $B$ and $C$; for instance, the class of all symmetric zero one matrices of order $n$ with $m^{2}$ ones, whenever $m^{2}$ is a multiple of $n$. The author is indebted to Dr. A. Ginsburg of the Technion for the following, less elementary, example:

$$
B=\left[\begin{array}{llll}
1 & 1 & 2 & 0 \\
1 & 1 & 2 & 0 \\
2 & 2 & 0 & 0 \\
0 & 0 & 0 & 4
\end{array}\right], \quad C=\left[\begin{array}{llll}
1 & 1 & 2 & 0 \\
1 & 1 & 2 & 0 \\
2 & 2 & 4 & 0 \\
0 & 0 & 0 & 0
\end{array}\right] .
$$




\section{BibliogRAPHy}

1. P. R. Beesack, Isoperimetric inequalities for the nonhomogeneous clamped rod and plate, J. Math. Mech. 8 (1959), 471-482.

2. P. R. Beesack and B. Schwarz, On the zeros of solutions of second order linear differential equations, Canad. J. Math. 8 (1956), 504-515.

3. R. Courant and D. Hilbert, Methods of mathematical physics, Vol. 1, Interscience, New York, 1953

4. G. H. Hardy, J. E. Littlewood and G. Polya, Inequalities, 2nd ed., University Press, Cambridge, 1952.

5. R. Jentzsch, Ueber Integralgleichungen mit positivem Kern, J. Reine Angew. Math. 141 (1912), 235-244.

6. S. Karlin, Positive operators, J. Math. Mech. 8 (1959), 907-937.

7. B. Schwarz, Bounds for the principal frequency of the nonhomogeneous membrane and for the generalized Dirichlet integral, Pacific J. Math. 7 (1957), 1653-1676.

8. F. Smithies, Integral equations, University Press, Cambridge, 1958.

9. F. G. Tricomi, Integral equations, Interscience, New York, 1957.

TEChNION-ISRAEL INSTITUTE OF TECHNOLOGY,

HAIFA, ISRAEL 